\title{
LES GAMMARIDÉS DES EAUX CONTINENTALES ET SAUMÂTRES DU SUD-EST DE LA RÉGION ARMORICAINE ET DU NORD DU BASSIN D'AQUITAINE
}

\author{
par \\ J. M. J. F. GRAS \& A. M. J. MAASEN \\ Adresse postale: Institut de Zoologie taxonomique (Zoölogisch Museum), Université d'Amsterdam, \\ Pays-Bas
}

\begin{abstract}
Fieldwork in French "départements" along the Atlantic coast from southern Brittany to the northern part of the Aquitaine Basin, revealed 324 inland and brackish localities with gammarids. Particularly interesting are 21 new stations of Echinogammarus spinulicornis (which was known from 3 places only, all more to the south). This species prefers the mid-course of rivers, especially habitats without vegetation.

Gammarus zaddachi is recorded for the first time south of the Loire.
\end{abstract}

Au cours des prospections exécutées pendant le mois d'avril 1970 dans les départements français bordant l'Océan Atlantique entre Lorient dans le Nord et Riberac dans le Sud, soit dit dans les départements du Morbihan, de la Loire Atlantique, des Deux-Sèvres, de la Charente-Maritime, de la Charente, et d'une partie des départements de la Vienne et de la Dordogne, 324 stations dans les eaux continentales ou saumâtres de Gammares furent relevées, en plus de 200 stations environ, où il n'y en avait pas. Les localités d'échantillonnage ont été illustrées dans la fig. 4.

Dans toutes les stations à Gammares, nous avons dosé certains ions importants $(\mathrm{Ca}, \mathrm{Cl})$, et noté le biotope (largeur et profondeur des eaux, température, intensité du courant, estimation du degré des pollutions, nature du fond, végétation...).

Gammarus pulex (Linnaeus, 1758) se trouve dans la plupart des échantillons. En deuxième lieu, en ce qui concerne l'abondance, se range Echinogammarus berilloni Catta, 1878. Moins abondants furent Gammarus chevreuxi Sexton, 1913, G. zaddachi Sexton, 1912, Echinogammarus spinulicornis Pinkster \& Stock, 1971 et Niphargus spec. I.

Le détail des échantillons figure dans le tableau

Il faut souligner l'absence de Gammarus duebeni Liljeborg, 1852, dans la région prospectée.
Cette espèce est connue du département du Finistère-Sud, où elle atteint apparemment la limite méridionale de sa distribution géographique.

\section{DISCUSSION DES ESPECES}

\section{Gammarus pulex}

Cette espèce est la plus abondante; elle a été trouvée partout. Souvent elle est le seul Gammaridé présent dans une station donnée, mais souvent aussi elle vit avec d'autres Gammares, surtout avec Echinogammarus berilloni.

Comme l'ont démontré Pinkster et al., 1970, Gammarus pulex et Echinogammarus berilloni sont parfaitement capables de coexister sans se concurrencer. Nos observations le confirment: dans 173 stations nous avons récolté uniquement $G$. pulex, et dans 25 stations uniquement $E$. berilloni tandis que les deux espèces coexistaient dans 87 stations.

Tableau I

Relevé des 324 échantillons d'Amphipodes nombre

G. pulex d'échantillons \%

G. pulex $+E$. berilloni $173 \quad 54,1$

$87-27,2$

G. pulex $+E$. berilloni + E. spinulicornis $\quad 13 \quad 4,1$

G. pulex + Niphargus spec. $\quad 2 \quad 0,6$

G. pulex + G. chevreuxi $\quad 2 \quad 0,6$

G. pulex + G. chevreuxi + E. berilloni 1

G. pulex +E. spinulicornis 110,3

E. berilloni

G. zaddachi

G. zaddachi + G. chevreuxi

G. chevreuxi

Niphargus spec.

E. spinulicornis

E. spinulicornis + E. berilloni

\begin{tabular}{rrr}
25 & 7,8 \\
3 & 1,0 \\
2 & 0,6 \\
1 & 0,3 \\
2 & 0,6 \\
2 & 0,6 \\
6 & 1,9 \\
\hline 320 & & 100,0
\end{tabular}

non-déterminables 


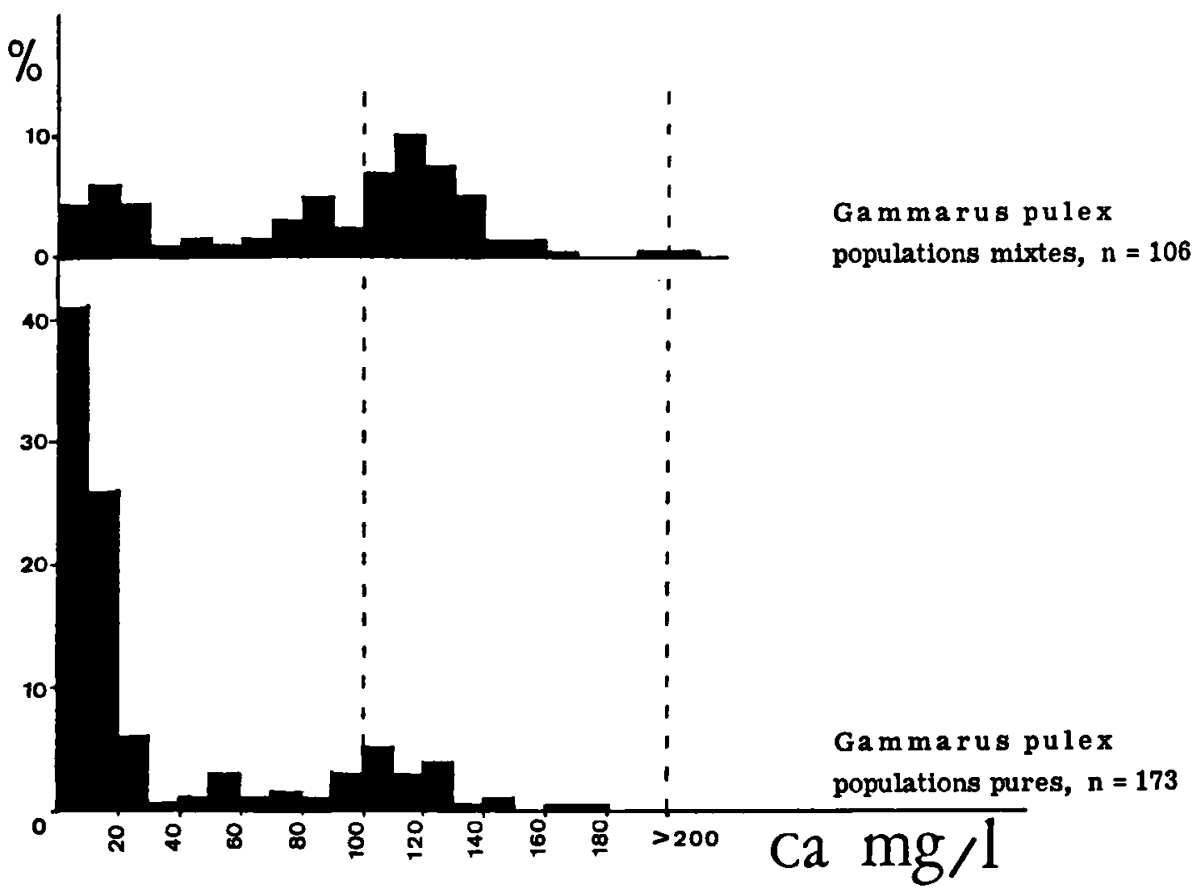

Fig. 1. La teneur en calcium de 173 stations de populations pures (en bas) et de 106 stations de populations mixtes de Gammarus pulex (en haut). Aux stations à G. pulex seul, les teneurs sont bien plus basses.

Parmi ces 173 stations à G. pulex, $67,0 \%$ montraient une teneur basse ou très basse en calcium, c'est-à-dire inférieure à $20 \mathrm{mg} / \mathrm{l} ; 15,0 \%$ seulement avaient entre 20 et $90 \mathrm{mg} \mathrm{Ca} / 1$, et le reste, soit $18,0 \%,>90 \mathrm{mg} / 1$.

Dans 106 stations, $G$. pulex était ensemble avec d'autres Gammares. La teneur en calcium de ces stations diffère considérablement de celles peuplées uniquement de $G$. pulex: les titres de 0-20 mg Ca / l se retrouvent en $13,7 \%$ des stations, la teneur de $20.90 \mathrm{mg} / 1$ en $26,4 \%$ des stations, de $>90 \mathrm{mg} / \mathrm{l}$ en $60,4 \%$. On constate un accroissement des teneurs en chaux dans les stations où vivent en commun les espèces différentes (voir également fig. 1).

La conclusion qu'on peut tirer de nos chiffres démontre que $G$. pulex est peu sensible à la dureté des eaux qu'il habite (comme l'ont déjà signalé d'autres auteurs), tandis que les autres espèces, surtout $E$. berilloni avec lequel il peut coexister sans se concurrencer, ont en général besoin d'une teneur en chaux plus élevée. Les besoins en calcium de ces espèces sont tels, qu'elles ne peuvent subsister dans les eaux avec une teneur en calcium inférieure à $20 \mathrm{mg} / \mathrm{l}$. En conséquence, les eaux titrant 0 à $20 \mathrm{mg} \mathrm{Ca} / 1$, forment une sorte de réservation pour $G$. pulex, à cause de son amplitude écologique plus large 1 ). Les tableaux IIa et IIb montrent que $G$. pulex recherche une région déterminée d'un cours d'eau. Ainsi ces relevés indiquent nettement la préférence de $G$. pulex pour des rivières de dimensions moyennes, autrement dit, pour le moyen-cours des rivières. La dominance de $G$. pulex dans ce type d'habitat a d'ailleurs été déjà signalée par d'autres auteurs.

G. pulex n'a pas une préférence très prononcée pour un microhabitat spécial. Le fond le plus favorable est celui constitué par des galets et du sable; cependant l'espèce forme également des populations importantes sur des fonds argileux ou sableux, le plus souvent dépourvus de végétation, mais aussi parmi les plantes aquatiques (voir tableau III).

1) Malheureusement, cette conclusion contraste fortement avec les résultats obtenus par Pinkster et al. (1970: 128) en Bretagne. Dans cette région granitique, $E$. berilloni comme $G$. pulex ont été trouvés en abondance dans des eaux avec une teneur basse ou très basse en calcium. Donc, tandis que nos propres observations semblent indiquer que la dureté des eaux joue une rôle importante, d'autres indications le contredisent. Nous ignorons en ce moment comment interpreter ces données si contradictoires. 


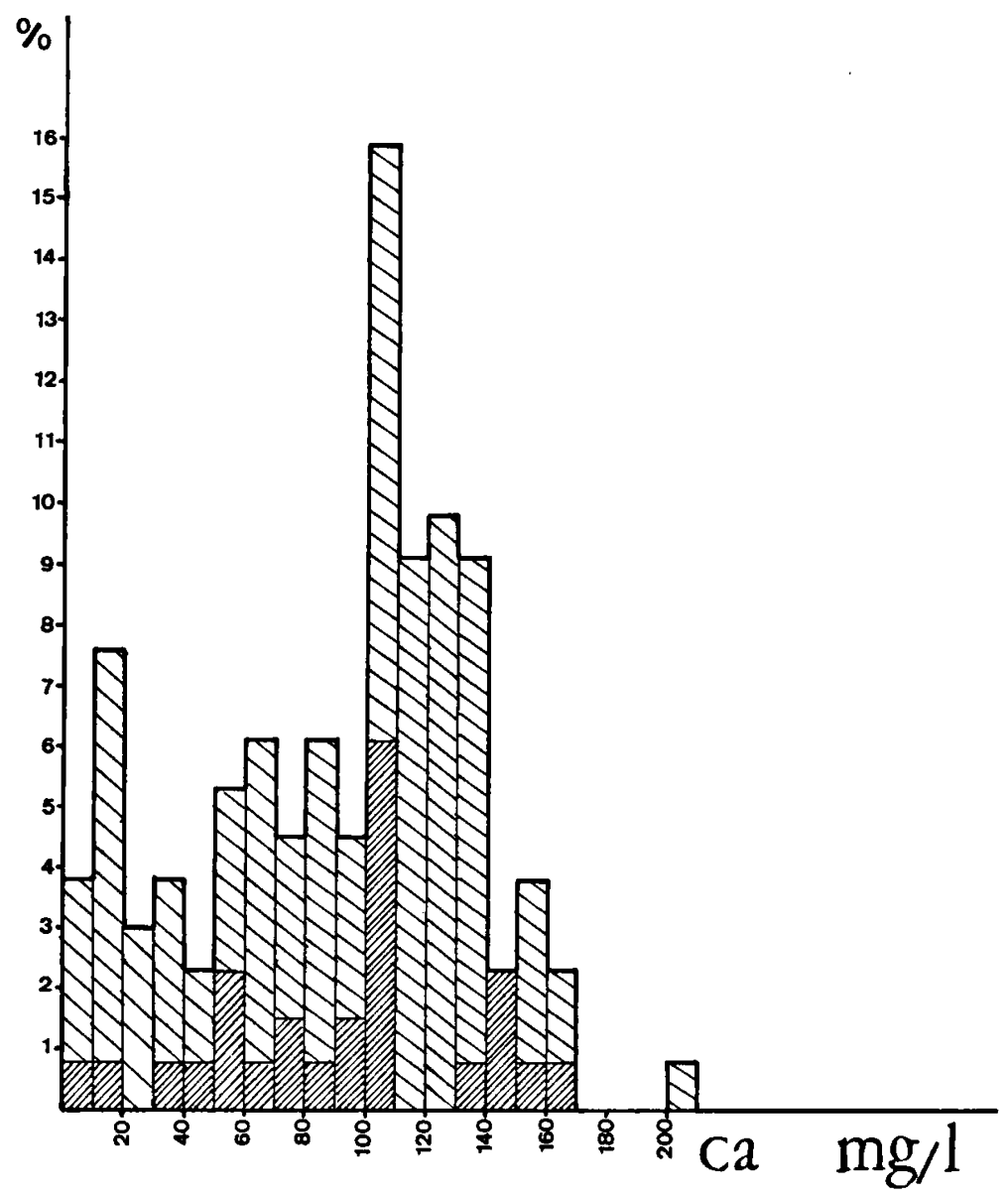

Fig. 2. La teneur en calcium de 112 stations d'Echinogammarus berilloni. Hachures étroites: populations pures $(\mathrm{n}=24)$. Hachures espacées: populations mélangées avec $G$. pulex $(\mathrm{n}=88)$.

G. pulex, espèce dominante des moyen-cours, peut subir sans grand danger un certain degré de pollutions de son milieu du type $\beta$-mésosaprobe (tableau IV).

\section{Echinogammarus berilloni}

Cette espèce est absente, ou virtuellement absente, dans la partie septentrionale de la région prospectée, donc dans les systèmes aquatiques de la Villaine et du cours inférieur de la Loire. Elle devient tout d'un coup abondante au Sud de la ligne Cholet - La Rochelle, donc dans le bassin de la Charente. $E$. berilloni a été trouvé dans 132 stations dont seulement 15 (soit $11,4 \%$ ), possédaient des eaux avec une teneur en calcium inférieure à $20 \mathrm{mg} / \mathrm{l} ; 41$ stations (soit $31,0 \%$ ) titraient de 20 à $90 \mathrm{mg} \mathrm{Ca} \mathrm{/} \mathrm{l;} \mathrm{le} \mathrm{reste,} 76$ stations $(57,6 \%)$, avait plus de $90 \mathrm{mg} / 1$. Dans la région prospectée, la préférence de cette espèce pour une

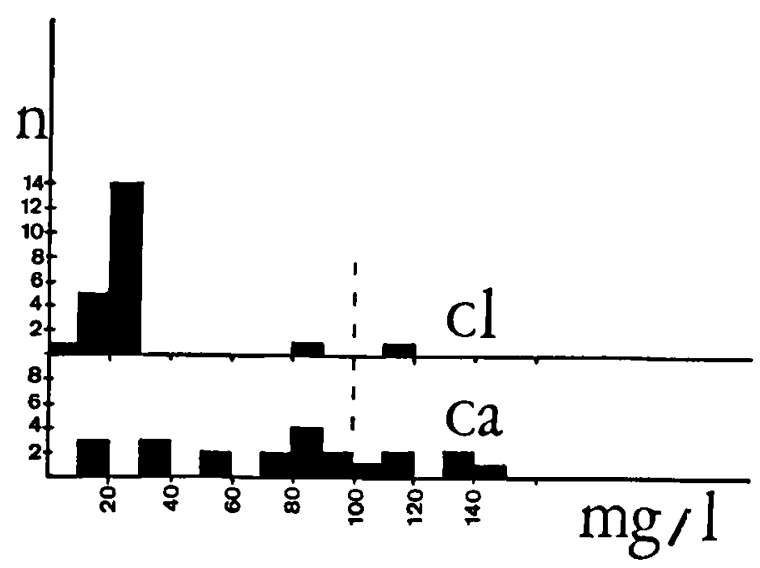

Fig. 3. La teneur en calcium (en bas) et en chlorure (en haut) de 22 stations d' Echinogammarus spinulicornis. Notez l'aspect de la courbe pour $\mathrm{Ca}$, entre $10 \mathrm{mg} / \mathrm{l}$ (eaux adoucies) jusqu'à $150 \mathrm{mg} / \mathrm{l}$ (eaux très minéralisées). 
Tableau II

a) Qualification des rivières habitées uniquement par Gammarus pulex

\begin{tabular}{|c|c|c|c|c|c|c|}
\hline largeur (m) & $0-0,50$ & $0,50-1$ & $1-2$ & $2-5$ & $>5$ & total \\
\hline \multicolumn{7}{|l|}{ profondeur (m) } \\
\hline $0-0,20$ & 13 & 17 & 10 & 5 & - & 45 \\
\hline $0,20-0,50 *)$ & 1 & 15 & 41 & 25 & 6 & 88 \\
\hline$>0,50$ & - & 一 & 5 & 17 & 13 & 35 \\
\hline total & 14 & 32 & 56 & 47 & 19 & 168 \\
\hline
\end{tabular}

b) Qualification des rivières habitées par Gammarus pulex avec d'autres espèces

\begin{tabular}{|c|c|c|c|c|c|c|}
\hline largeur (m) & $0-0,50$ & $0,50-1$ & $1-2$ & $2-5$ & $>5$ & total \\
\hline \multicolumn{7}{|l|}{ profondeur (m) } \\
\hline $0-0,20$ & 2 & 4 & 5 & 8 & 一 & 19 \\
\hline $0,20-0,50 *)$ & - & 2 & 19 & 30 & 9 & 60 \\
\hline$>0,50$ & - & - & 2 & 8 & 11 & 21 \\
\hline total & 2 & 6 & 26 & 46 & 20 & 100 \\
\hline
\end{tabular}

*) Profondeur à l'endroit d'échantillonnage, mais non la profondeur maxima de la rivière.

\section{Tableau III}

Nature des fonds des stations à Gammarus pulex et (entre parenthèses) d Echinogammarus berilloni

\begin{tabular}{lrccccccc}
\multicolumn{1}{c}{ fond } & argile & $\begin{array}{c}\text { argile } \\
\text { sable }\end{array}$ & $\begin{array}{c}\text { sable }+ \\
\text { humus }\end{array}$ & $\begin{array}{c}\text { sable }+ \\
\text { vase }\end{array}$ & $\begin{array}{c}\text { sable } \\
\text { pur }\end{array}$ & $\begin{array}{c}\text { sable }+ \\
\text { galets }\end{array}$ & galets & total \\
\hline $\begin{array}{l}\text { végétation } \\
\text { plantes aquatiques }\end{array}$ & $15(3)$ & $6(-)$ & $-(-)$ & $4(3)$ & $10(8)$ & $32(15)$ & $7(3)$ & $74(32)$ \\
$\begin{array}{l}\text { plantes aquatiques } \\
\text { et palustres }\end{array}$ & $3(1)$ & $1(-)$ & $-(-)$ & $-(-)$ & $12(9)$ & $8(4)$ & $-(-)$ & $24(14)$ \\
$\begin{array}{l}\text { plantes palustres } \\
\text { nulle }\end{array}$ & $\mathbf{8 ( 1 )}$ & $3(3)$ & $1(-)$ & $2(2)$ & $16(10)$ & $19(5)$ & $3(4)$ & $52(25)$ \\
\hline total & $21(4)$ & $14(2)$ & $-(1)$ & $-(-)$ & $12(8)$ & $56(25)$ & $21(14)$ & $124(54)$ \\
\hline
\end{tabular}

Tableau IV

Pollution du milieu de G. pulex (entre parenthèses celle de E. berilloni)

normal

(oligosaprobe)

légèrement

pollué (oligo- B-mésosaprobe)

nombre d'

échantillons
$40(28)$

$209(90)$ moyennement pollué

( $\beta$-mésosaprobe)

30 (14) total

279 (132)

Tableau V

Qualification des rivières habitées par Echinogammarus berilloni

\begin{tabular}{lcccccc}
\multicolumn{1}{c}{ largeur $(\mathrm{m})$} & $0-0,50$ & $0,50-1$ & $1-2$ & $2-5$ & $>5$ & total \\
\hline profondeur $(\mathrm{m})$ & & & 3 & 6 & 16 & -14 \\
$0-0,20$ & - & 3 & 26 & 40 & 14 & 29 \\
$\left.0,20-0,50^{\star}\right)$ & - & - & 2 & 20 & 11 & 83 \\
$>0,50$ & 4 & 6 & 34 & 76 & 25 & 145
\end{tabular}

*) Profondeur à l'endroit d'échantillonnage, mais non la profondeur maxima de la rivière. 


$$
\text { (1) }
$$

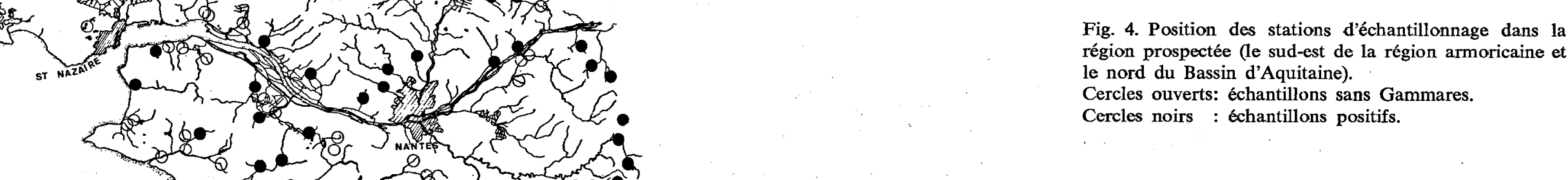

Fig. 4. Position des stations d'échantillonnage dans la sud-est de la région armoricaine et le nord du Bassin d'Aquitaine).

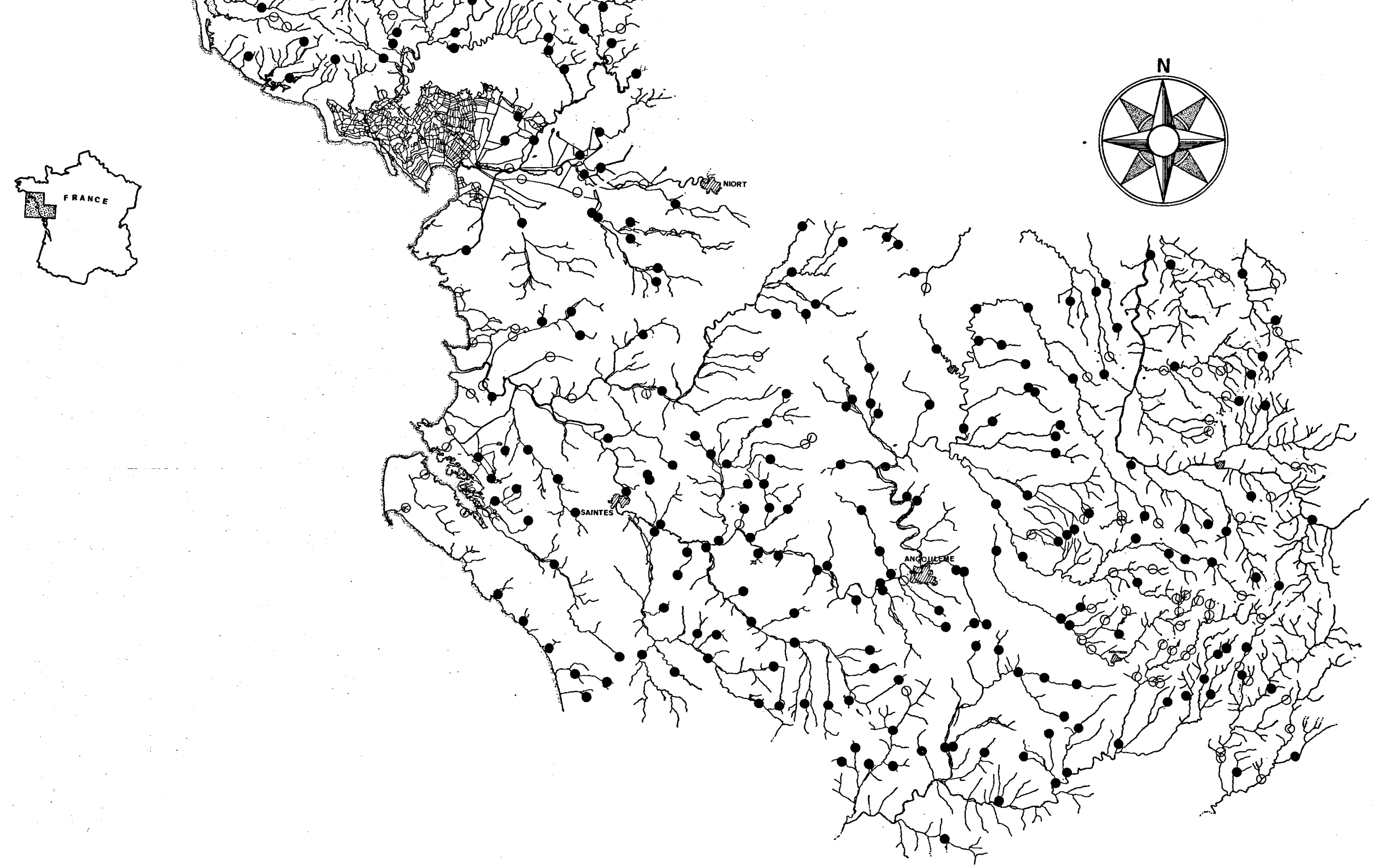

i. 206

y 


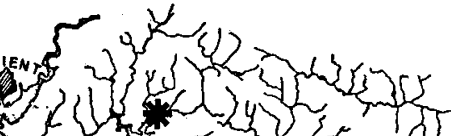

(n)

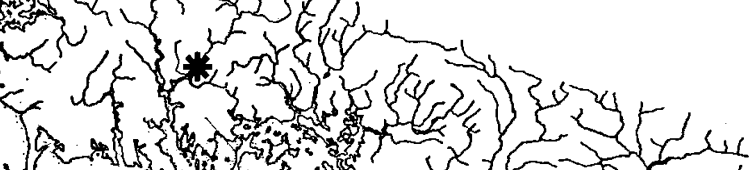

(n)
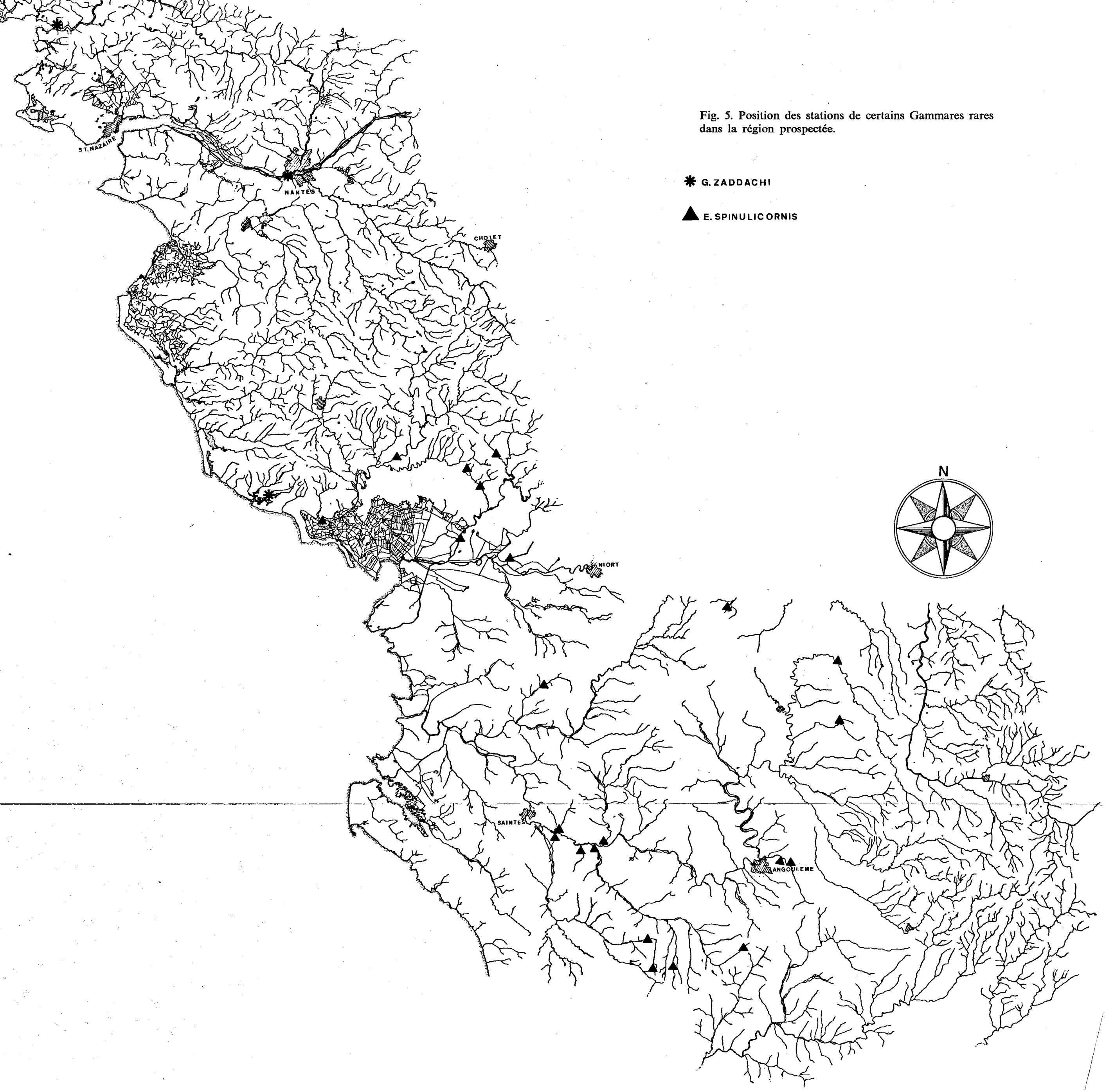
Tableau VI

Localités nouvelles d'Echinogammarus spinulicornis

\begin{tabular}{|c|c|c|c|c|c|c|}
\hline $\begin{array}{l}\text { no. } \\
\text { station }\end{array}$ & département & route no. & $\begin{array}{c}\text { nom du cours } \\
\text { d'eau }\end{array}$ & lieu le plus proche & position générale & $\begin{array}{l}\text { jour } \\
\text { (avril } \\
1970)\end{array}$ \\
\hline J 103 & la Vendée & N 747 & sans nom & Ville d'Angers & $21 \mathrm{~km} \mathrm{O} \mathrm{de} \mathrm{Lucon}$ & 13 \\
\hline J 105 & la Vendée & D 7 & Lay & Moutiers-sur-le Lay & $\begin{array}{l}6 \mathrm{~km} \text { NE de Moreuil- } \\
\text { sur-Lay }\end{array}$ & 13 \\
\hline J 115 & la Vendée & D 104 & sans nom & Mon Repos & $\begin{array}{l}7 \mathrm{~km} \text { NO de Fontenay- } \\
\text { le Comte }\end{array}$ & 13 \\
\hline J 116 & la Vendée & D 66 & sans nom & L'Hermenault & $\begin{array}{l}14 \text { km NO de Fontenay- } \\
\text { le Comte }\end{array}$ & 13 \\
\hline J 118 & la Vendée & D 63 & sans nom & Cézais & $\begin{array}{l}13 \text { km N de Fontenay- } \\
\text { le Comte }\end{array}$ & 14 \\
\hline J 123 & la Vendée & D 25 & Vendée & Le Gué de Velluive & $\begin{array}{l}14 \text { km SO de Fontenay- } \\
\text { le Comte }\end{array}$ & 14 \\
\hline J 127 & la Vendée & D 25 & $\begin{array}{l}\text { Canal de la } \\
\text { Vieille Autisse }\end{array}$ & Grande Bernegoue & $\begin{array}{l}18 \text { km S de Fontenay- } \\
\text { le Comte }\end{array}$ & 15 \\
\hline J 131 & les Deux-Sèvres & D 15 & sans nom & Lezay & $11 \mathrm{~km} \mathrm{NE}$ de Melle & 15 \\
\hline J 135 & Vienne & D 107 & Charente & Charroux & $8 \mathrm{~km}$ E de Ovray & \\
\hline J 150 & Charente & D 176 & L'Or & Champagne Moulon & $20 \mathrm{~km} \mathrm{E}$ de Ruffec & 16 \\
\hline J 188 & Charente & N 737 & Charente & La Chapelle & $6 \mathrm{~km} \mathrm{SE}$ de Aigre & 19 \\
\hline J 207 & Charente-Mar. & D 212-e4 & Frézence & Ligueuil & $\begin{array}{l}16 \text { km N de St. Jean } \\
\text { d'Angely }\end{array}$ & 21 \\
\hline J 239 & Charente-Mar. & D 136 & Gua & Salignac & $10 \mathrm{~km} \mathrm{O}$ de Cognac & 23 \\
\hline J 240 & Charente-Mar. & D 128 & sans nom & St. Séver & $11 \mathrm{~km}$ SE de Saintes & 23 \\
\hline J 241 & Charente-Mar. & D 24 & sans nom & Orlac & $13 \mathrm{~km}$ SE de Saintes & 23 \\
\hline J 242 & Charente & N 732 & Canal du Né & Merpins & 8 km O de Cognac & 23 \\
\hline J 243 & Charente & D 83 & Antenne & Jarnouzeau & $4 \mathrm{~km} \mathrm{O}$ de Cognac & 23 \\
\hline J 299 & Charente & D 38 & Trèfle & Xandeville & $4 \mathrm{~km} \mathrm{O}$ de Barbezieux & 28 \\
\hline J 301 & Charente & D $14 / \mathrm{N}$ & Trèfle & Reignac & 8 km S de Barbezieux & 28 \\
\hline J 302 & Charente & D 58 & Beau & Condéon & $8 \mathrm{~km}$ SE de Barbezieux & 28 \\
\hline J 307 & Charente & D 54 & sans nom & Puypéroux & $23 \mathrm{~km} \mathrm{~S}$ de Angoulême & 28 \\
\hline
\end{tabular}

Tableau VII

Qualification des rivières habitées par Echinogammarus spinulicornis

\begin{tabular}{|c|c|c|c|c|c|c|}
\hline largeur $(\mathrm{m})$ & $0-0,50$ & $0,50-1$ & $1-2$ & $2-5$ & $>5$ & total \\
\hline \multicolumn{7}{|l|}{ profondeur (m) } \\
\hline $0-0,20$ & 1 & - & 2 & 一 & - & 3 \\
\hline $0,20-0,50 *)$ & 一 & 1 & 3 & 5 & 4 & 13 \\
\hline$>0,50$ & 一 & - & 1 & 2 & 3 & 6 \\
\hline total & 1 & 1 & 6 & 7 & 7 & 22 \\
\hline
\end{tabular}

*) Profondeur à l'endroit d'échantillonnage, mais non la profondeur maxima de la rivière.

Tableau VIII

Nature des fonds des 22 stations d'Echinogammarus spinulicornis

\begin{tabular}{|c|c|c|c|c|c|c|c|c|}
\hline fond & argile & $\begin{array}{c}\text { argile }+ \\
\text { sable }\end{array}$ & $\begin{array}{l}\text { sable } \\
+ \text { vase }\end{array}$ & $\begin{array}{l}\text { sable }+ \\
\text { humus }\end{array}$ & $\begin{array}{l}\text { sable } \\
\text { pur }\end{array}$ & $\begin{array}{c}\text { sable }+ \\
\text { galets }\end{array}$ & galets & total \\
\hline $\begin{array}{l}\text { végétation } \\
\text { plantes aquatiques }\end{array}$ & - & - & - & - & - & - & - & 0 \\
\hline $\begin{array}{l}\text { plantes aquatiques } \\
\text { et palustres }\end{array}$ & - & - & - & - & - & 1 & - & 1 \\
\hline plantes palustres & 1 & 1 & 1 & - & 2 & 2 & - & 7 \\
\hline nulle & - & 2 & - & 1 & 2 & 8 & 1 & 14 \\
\hline total & 1 & 3 & 1 & 1 & 4 & 11 & 1 & 22 \\
\hline
\end{tabular}


Tableau IX

Pollution du milieu à Echinogammarus spinulicornis

\begin{tabular}{cccc}
$\begin{array}{c}\text { normal } \\
\text { (oligosaprobe) }\end{array}$ & $\begin{array}{c}\text { légèrement pollué } \\
\text { (oligo- } \\
\beta \text {-mésosaprobe) }\end{array}$ & $\begin{array}{c}\text { moyennement } \\
\text { pollué } \\
(\beta \text {-mésosaprobe) }\end{array}$ & total \\
3 & 15 & 4 & 22 \\
\hline
\end{tabular}

dureté d'eau élevée est donc nettement prononcée (la situation en Bretagne est différente, voir note inf rapaginale 1).

Le tableau $\mathrm{V}$ montre sa préférence pour le moyen-cours d'une rivière, tandis que la nature du fond des stations habitées par $E$. berilloni est représentée dans le tableau III. Il est donc évident que ses préférences pour un certain genre de fond ou pour une certaine végétation sont aussi peu notables, voire plus ou moins identiques à celles de $G$. pulex, espèce avec laquelle $E$. berilloni coexiste d'ailleurs. Comme G. pulex, E. berilloni peut supporter un certain degré de pollutions dans son milieu du type $\beta$-mésosaprobe (voir tableau IV).

\section{Echinogammarus spinulicornis}

Jusqu'ici cette espèce n'était connue que de trois localités (Pinkster \& Stock, 1971), dont les Sources de la Touvre (près de Ruelle, à l'Est d'Angoulême) étaient la plus septentrionale. Nous l'avons rencontrée dans 21 stations nouvelles, énumérées dans le tableau VI, et dans sa localité classique (Sources de la Touvre), soit au total dans 22 stations. Dans deux de ces stations (soit $9,0 \%$ du total), $E$. spinulicornis était seul, en une station $(4,5 \%)$ il était ensemble avec $E$. berilloni, tandis qu'en 13 stations même $(59,0 \%)$, il vivait en commun avec $G$. pulex et $E$. berilloni. Ceci indique déjà nettement, que les préférences écologiques d' $E$. spinulicornis sont plus ou moins identiques à celles des espèces coexistantes. Ceci est confirmé par l'étude du tableau VII; on peut en conclure que l'espèce habite surtout le moyen-cours des rivières.

Quant à la nature du fond (tableau VIII), E. spinulicornis montre une préférence distincte par rapport aux $G$. pulex et $E$. berilloni. Il est plus abondant dans un habitat sans aucune végétation, et il est peu commun parmi une végétation palustre. Comme G. pulex et E. berilloni, E. spinulicornis supporte un certain degré de pollutions dans son milieu du type $\beta$-mésosaprobe (tableau IX).

Les courbes des teneurs en calcium et en chlore (fig. 3) aux stations d'échantillonnage ne montrent rien de particulier. Comme pour toute espèce dulcicole, 20 des 22 stations avaient une chlorinité inférieure à $30 \mathrm{mg} \mathrm{/} 1$. Quant à la teneur en chaux, on voit une distribution uniforme entre 10 et 150 mg Ca / l. En tout cas, il est évident que l'espèce n'est pas confinée aux eaux fortement minéralisées; elle diffère à cet égard de l'espèce qui en est systématiquement la plus proche, $E$. pungens $(H$. Milne Edwards), qui habite toujours un milieu avec une haute teneur en ions (eaux minéralisées,estuaires, lagons salés).

\section{Gammarus chevreuxi}

Nous avons trouvé cette espèce dans six localités légèrement saumâtres (47 - $630 \mathrm{mg} \mathrm{Cl} / \mathrm{l})$. Parmi ces six stations, deux étaient peuplées par $G$. chevreuxi avec $G$. zaddachi, un groupement d'espèces qui est normale dans un milieu un peu salin. Il faut insister sur trois autres stations, où $G$. chevreuxi coexistait avec $G$. pulex et (dans un cas) avec cette espèce et avec $E$. berilloni. Une telle coexistence n'est possible que dans une zone étroite de transition entre les domaines limnique et saumâtre. Là, au moment d'échantillonnage, la chlorinité titrait entre 47 et $108 \mathrm{mg} / \mathrm{l}$.

Les six localités où nous avons rencontré $G$. chevreuxi, sont toutes dans l'aire de distribution connue de cette espèce (de Bretagne et de la partie Sud de l'Angleterre jusqu'à la côte atlantique du Maroc) et nos analyses de son milieu physique n'ajoutent rien de nouveau à son autécologie.

\section{Gammarus zaddachi}

C'est une espèce des eaux saumâtres, que nous n'avons rencontrée qu'en cinq localités. D'après nous, elle paraît atteindre la limite méridionale de sa distribution géographique ${ }^{2}$ ) dans la région que nous venons de prospecter. La station plus au Sud connue jusqu'ici est à notre connaissance Nantes,

2) G. salinus Spooner, 1947 (considéré dans le passé comme sous-espèce de zaddachi, mais incontestablement une espèce distincte, interstérile) s'étend plus loin vers le Sud. 
au bord de la Loire, 2 novembre 1891 (collection $\mathbf{E}$. Chevreux, Muséum National d'Histoire Naturelle, Paris; voir Sexton, 1942: 583). Nos récoltes renfermaient un seul échantillon pris au Sud de la Loire, à savoir à $4 \mathrm{~km} \mathrm{S.E.} \mathrm{de} \mathrm{Talmont,} \mathrm{dép.} \mathrm{de}$ la Vendée, près du pont, sur la route départementale D 21 (chlorinité $1066 \mathrm{mg} /$ 1). Les quatre autres stations sont au Nord de la Loire, donc, à l'intérieur de l'aire de distribution connue.

\section{REMERCIEMENTS}

Tous nos remerciements vont aux Professeur J. H. Stock et M. S. Pinkster (d'Amsterdam) pour leur assistance précieuse durant tout notre travail. Nous exprimons également notre gratitude à l'Université d'Amsterdam, qui a subventionné notre travail sur le terrain, dans le cadre d'une thèse 3 ème cycle.

\section{REFERENCES BIBLIOGRAPHIQUES}

Pinkster, S., A. L. Dennert, B. Stock \& J. H. Stock, 1970. The problem of European freshwater populations of Gammarus duebeni Liljeborg, 1852. Bijdr. Dierk., 40 (2) : 116-147.

Pinkster, S. \& J. H. Stock, 1971. Sur deux Echinogammarus limniques nouveaux du Bassin d'Aquitaine.
Bijdr. Dierk., 41 (1) : 37-51.

Sexton, E. W., 1942. The relation of Gammarus zaddachi Sexton to some other species of Gammarus occurring in fresh, estuarine and marine waters. J. mar. biol. Ass. U.K., 25 : 575-606.

Reçu: le 5 Octobre 1970 